\title{
Design of Gripper for Special Manipulator of Milk Machine
}

\author{
Kong Jiali $^{1,2, a}$, Ning Tingzhou ${ }^{1,2, b}$ and Zhang Weiwei ${ }^{1,2, c}$ \\ ${ }^{1}$ School of Mechanical Engineering, Shandong Huayu University of Technology, Dezhou 253034, China \\ ${ }^{2}$ College of Engineering, China Agriculture University, Beijing 100083, China \\ a kongjialiskd@126.com, ${ }^{\text {b }}$ ning-tingzhou@163.com, ${ }^{\text {c } z h a n g 7412860 @ 163 . c o m ~}$
}

Keywords: manipulator, gripper, structure

\begin{abstract}
By analyzing the working environment and mode of manipulator, the structure of manipulator was designed, which has four shafts, the upper and lower shaft, the shaft of rotation, the telescopic shaft, and the rotation shaft. The main structure of manipulator was divided into three parts, which are base part, the four shaft part, and the hand part. A vacuum sucker is mounted on the gripper to absorb the workpiece. Experiments showed that the velocity of manipulator are fast, stable without trembling.
\end{abstract}

\section{Introduction}

Manipulator is mainly used in labor-intensive processing industry, instead of manual to complete monotonous repeated work, improve productivity and product pass rate [1]. As a production tool with higher intelligence, manipulator has been widely used and developed in recent years [2]. The application of manipulator expands the function of human limbs and brain, and avoids dangerous, harmful, low temperature and high fever work. The arm is connected to the bottom and middle of the hand, and there is no joint arm and cross section arm [3]. The manipulator consists of a base, shoulder, arm, elbow, wrist and gripper [4]. The bottom cover of the soybean milk maker is a cylindrical and light object, about $0.2 \mathrm{~kg}$. It can be picked up with a very small force. In order to prevent scratching the surface, a vacuum sucker is used to pick it up. The surface of the object is smooth, and the sucker fits well with the surface of the object. The layout of the sucker has an important influence on the stability and safety of the clamping workpiece [5]. The forces between the suckers will deviate from each other if the arrangement is not reasonable, which can generate torque and bending moment, make the workpiece skew when clamping. There is a risk of sliding during operation when it does not place on the correct position of the mould, which may lead to production accidents.

\section{Design of gripper for gripper}

\subsection{Methods of obtaining vacuum by sucker}

The methods of obtaining vacuum by sucker are mainly as follows, the squeezing and exhausting method, the vacuum method through vacuum pump and the airflow negative pressure method. The squeezing and exhausting method needs to apply certain external force in the sucker to realize. The external force is small and may produce less adsorption, thus, the workpiece is not tight while the external force will damage the workpiece. So it is very difficult to grasp the external force. This method was not suitable. The vacuum method goes through vacuum pump: in order to absorb the workpiece, the vacuum pump is used to pump the air through the vacuum valve to draw the air from the sucker's cavity to form a negative pressure. This method requires a vacuum pump, which increases the burden of the equipment, and because the workpiece is light, there is no need for greater negative pressure. This method was neither adaptive. The airflow negative pressure method is: in order to make the suction disk produce negative pressure to absorb the workpiece, it can be mainly achieved by the opening of the control valve, then the flowing air can produce the high speed jet in the trachea. This kind of method is quite easy to 
realize, only adds one vacuum valve in the air path. The generating negative pressure is also relatively stable, so this method was adequate. Through the analysis of the above three methods of obtaining vacuum, the third method was adopted for the sucker of the manipulator.

\subsection{Selection of vacuum sucker}

Selection method of vacuum sucker:

1) determining the diameter of the sucker according to the weight of the workpiece.

2) determining the diameter of the sucker and the number of suckers according to the area of the workpiece.

3) determining the material and style of the sucker according to the material and shape of the workpiece.

The method of selecting the sucker by experience, the conservative calculation method of suction force of the sucker is the $\mathrm{cm} 2$ of the radius of the sucker, that is the kilogram numbers of the suction force.

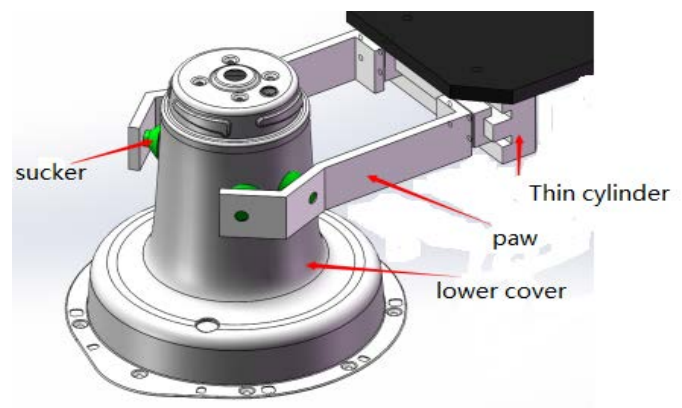

Figure 1 Schematic diagram of hand claw grabbing workpiece

The working status of the manipulator clamping piece(the bottom cover of the soybean milk maker) is shown in figure 1.Calculation conditions, the outer diameter of the clamping part of the bottom cover is $70 \mathrm{~mm}$ and the weight is $0.2 \mathrm{~kg}$. The primary election diameter of the sucker is 2 $\mathrm{cm}$. Calculating the suction force of sucker is

$$
F=\frac{S P}{\mu}
$$

Where $S$ is the surface area of the sucker, $P$ is the air pressure, $\mu$ is the safety factor, generally takes up to 2.5. According to the sucker sample supplied by some sucker companies, the vacuum degree of the sucker is $-750 \mathrm{mbar}(0.75 \mathrm{~kg} / \mathrm{cm} 2)$, the diameter of the sucker is $2 \mathrm{~cm}$, the safety factor is 3 . It is concluded that F equals to $0.785 \mathrm{~kg}$. Four same suckers are chosen, so the total suction force of the manipulator is $3.14 \mathrm{Kg}$. It is much larger than the weight of the workpiece, which can completely meet the grasping conditions. Another advantage of the choice of the sucker with a large surface area is that the large contact area can maintain good stability. As shown in Figure 1, the four suckers are installed by a symmetrical distribution method. The two suckers have an angle of 60 degrees, which can be well supported and automatically aligned.

\subsection{Structure design of gripper}
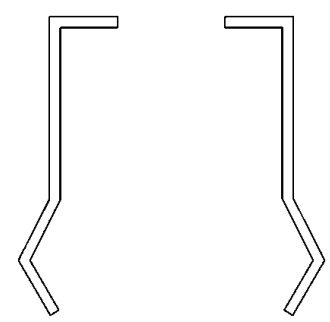

Figure 2 Structure of gripper

The structure of the gripper is shown in Figure 2. It is made up of $5 \mathrm{~mm}$ thick stainless steel. It has high strength and stiffness, so it can withstand large and frequent impact. The surface is not 
rusty, so there is no rust and other foreign objects falling down when the workpiece is clamped, which can meet the requirement of the health.

\subsection{Cylinder selection of gripper}

The slider finger cylinder was chosen. The volume of the cylinder is smaller than that of the ordinary cylinder. There is a linear guide rail in the middle and two sliding rails slide on the guide rail. The structure not only occupies less space, but also can withstand large torque, and it has quick sensitive and high precision. A magnetic switch is installed on the cylinder to detect the opening and closing of the hand claw [6][7]. The selected model is MHF2-12D2. Its type represents 2 hand claws, $12 \mathrm{~mm}$ cylinder diameter, double-acting cylinder, \pm 0.05 repetition accuracy, 48mm long route. The slider finger cylinder is shown in Figure 3 . The main body parameters of manipulator are shown in Table 1.

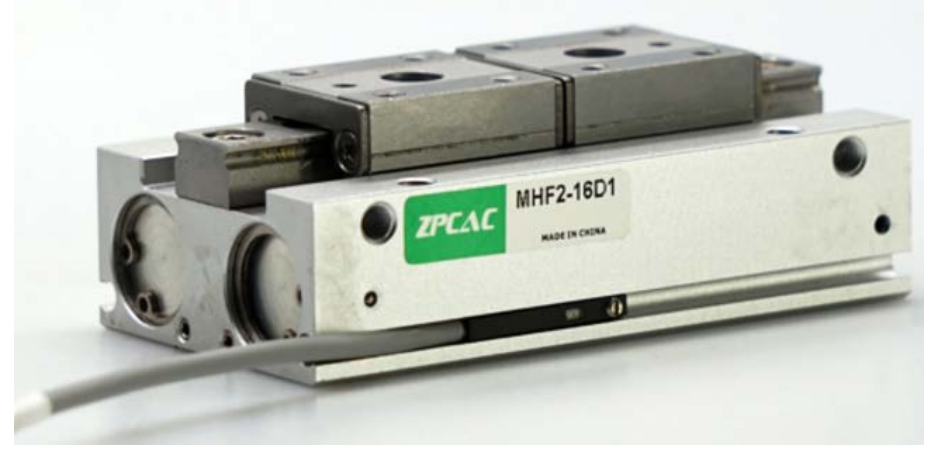

Figure3 Slide finger cylinder

Table 1 Main body parameters of manipulator

\begin{tabular}{|c|c|}
\hline Parameters & Values \\
\hline $\mathrm{Load} / \mathrm{kg}$ & 5 \\
\hline Positional accuracy/mm & 0.5 \\
\hline
\end{tabular}

\section{Conclusions}

The structure of manipulator is designed according to the size and shape of the gripping workpiece. The vacuum of the sucker was obtained by the airflow negative pressure method. The size, number and distribution of the sucker are calculated according to the weight of the workpiece. The gripper satisfies to the requirement of clamping the bottom cover of soybean milk machine. It provides a more secure and stable working environment for grass-roots operators.

\section{References}

[1] Mei Xuechuan. (2012) Development and Research of stamping equipment loading and unloading Robo. Guangzhou: South China University of Technology.

[2] Yang Zhilong. (2015) Research on Modeling Design of Intelligent Mechanical arm. Chinese machinery, 3, 63-64.

[3] Fan Xiaolan, Zhao Chunfeng. (2012) The manipulator based on PLC control in the implementation of the MCGS. Manufacturing automation, 6-8,25.

[4] Li Xiaochen, Liu Ziyang, Li Deyuan. (2014) Design and Research of six degrees of Freedom underwater manipulator. Hydraulic and pneumatic,3,63-64.

[5] Guan Ming, Zhou Xilun, Ma Lijing. (2012) Design of manipulator control system based on PLC. Coal mine machinery, 120-121,142.

[6] Zhang Yonglin. (2004) Research and Design of Pneumatic System in Full Packing Maching 
for Cranular Material. Hydraulic and pneumatic, 11, 33-34.

[7] Zhou Qi, Wu Wanzhong, Wu Gang. (2013)Design and manufacture of multifunction Cam manipulator. Industry and Technology Forum, 19, 55-56. 